- ACORN Australan college of | JOURNAL OF PERIOPERATIVE NURSING

\title{
The future of nurse education in Australia and implications for perioperative nursing
}

Follow this and additional works at: https://www.journal.acorn.org.au/jpn

Part of the Health Services Administration Commons, Health Services Research Commons, Perioperative, Operating Room and Surgical Nursing Commons, and the Surgery Commons

c) (i)

This work is licensed under a Creative Commons Attribution 4.0 License.

\section{Recommended Citation}

Duff, Jed (2020) "The future of nurse education in Australia and implications for perioperative nursing," Journal of Perioperative Nursing: Vol. 33 : Iss. 1 , Article 1.

Available at: https://doi.org/10.26550/2209-1092.1075

https://www.journal.acorn.org.au/jpn/vol33/iss1/1

This Article is brought to you for free and open access by Journal of Perioperative Nursing. It has been accepted for inclusion in Journal of Perioperative Nursing by an authorized editor of Journal of Perioperative Nursing. 


\section{ACORN}

The Australian College of Perioperative Nurses (ACORN) is a registered Australian company and health promotion charity. It exists to serve its members, the perioperative profession, the patient and the community to promote the prevention and control of disease.

ACORN's mission is to advance safe, quality perioperative nursing care for Australians.

\section{ACORN Board of Directors}

Garry Stratton

President

Trent Batchelor

Director

Patricia Flood

Director

Karen Hay

Director

Conference Committee Chair

Grace Loh

Director

Paula Foran

Member Director

Sophie Ehrlich

Director

Donna Stevens

Director

\section{The future of} nurse education

Associate Professor Jed Duff PhD, RN

Journal Editor

\section{in Australia and implications for perioperative nursing}

Recently there has been a national review into nurse education in Australia conducted by Emeritus Professor Steven Schwartz on behalf of the federal government. The aim was to examine the current educational preparation of Australian nurses to ensure the profession is adequately equipped to meet the future needs of the nation's evolving health system. The review was shaped by extensive consultations across Australia, with more than 1100 educators, clinicians, supervisors, policymakers, patient groups, students, managers and unions providing feedback. I think it is appropriate to review the report here because of the importance, as ACORN stated in its most recent strategic plan, of influencing the nursing curriculum to ensure a greater focus on perioperative care. Below, I outline some of the key recommendations from the report and their potential implications for perioperative nursing.

Perhaps the most contentious of the recommendations is that all candidates for registration should sit a national exam. The exam would consist of a literacy and numeracy assessment as well as an outcomes-based cognitive and behavioural assessment. The Nursing and Midwifery Board of Australia (NMBA) is currently rolling out such an exam for international applicants, and the Commissioner felt it would be equitable to include domestic applicants. As I see it, this exam would act as a proxy national curriculum as all schools of nursing would be forced to teach to it. Whether you like this idea or not, it would help provide some sorely needed consistency in the educational preparation of new registered nurses (RNs) in Australia. For ACORN, it would also provide an opportunity to influence the questions on the exam, thus ensuring core perioperative nursing knowledge is taught and examined.
The preparation of new graduate nurses and their transition into the workforce was a significant focus of the recommendations. I am sure that during the consultation process, the Commissioner heard many people complain that graduates were not 'work-ready'. Reading between the lines of the report, it would seem that he felt the profession was not entirely in agreement on what this entailed. The report recommends that the NMBA practice standards list the specific core knowledge, skills and procedural competencies new RNs need in order to function in any workplace setting. The development of this list provides ACORN with an opportunity to lobby for the inclusion of knowledge, skills and procedural competencies central to perioperative nursing practice.

The report identified that clinical placement is central to nursing education; however, it was noted that there is limited evidence on what this should comprise. After benchmarking with comparable international health 
systems, the Commissioner made the recommendation to increase the minimum number of clinical placement hours from 800 to 1000. It was acknowledged that finding these extra hours will be a challenge since it is already difficult to find quality placements for nursing students - expansion of rural and nontraditional placements areas was proposed as one potential solution. Increasing clinical placement opportunities for nursing students in the perioperative environment is the perfect opportunity for our specialty to influence undergraduate education. To do this, however, many departments will need to overcome their insistence on only taking one or two students at a time and only students in their third year.

The quality of clinical placements and clinical education for students and new graduates was a focus of the report. The report noted that due to the sheer number of students and the pressure to find placements, the quality of the experience was sometimes compromised. It was noted that RN training is a collaboration between education providers and the health care industry but these two groups do not necessarily work well together. The report urged better coordination between the two sectors and highlighted the Victorian Department of Health and Human Services Best Practice Clinical Learning Environment as an exemplary framework. The clinical education of new graduate nurses was identified as another challenge that could be addressed with better coordination. The report recommended the establishment of a national webbased Transition to Practice program that was compulsory for all nurses in their first year.

ACORN is promoting a greater emphasis on perioperative nursing in the undergraduate curriculum just as many of the other nursing specialty groups are. Unfortunately for us, some specialty groups are better at having their voice heard by government. From my experience, the groups who traditionally have the ear of the federal government are those advocating for mental health nurses, nurse practitioners, health informatics, rural and remote health services, Aboriginal and Torres State islanders and primary health care. Unsurprisingly, there are recommendations in the report specific to each of these six areas with a particular emphasis on the need to focus on public health issues. Given this focus, ACORN should emphasise the view of the World Health Organization that surgery is an essential public health intervention that plays a critical role in the prevention and treatment of disease and disability.

As you can imagine, schools of nursing are continually adapting the curriculum to meet the needs of the health system. However, there are a limited number of hours available in a three-year degree so priorities need to be set. Without a national curriculum or national exam, universities are free to set these priorities themselves and currently, in many schools, perioperative nursing is not being viewed as essential. The report recommends exploring ways to extend the nursing degree. A suggestion to be explored is a nested course which comprises a two-year diploma leading to registration as an enrolled nurse (EN) followed by a two-year degree leading to registration as an RN. An extended undergraduate program has many challenges, but it does offer the prospect of space to include perioperative nursing content currently curtailed by time limits.

I have only mentioned a number of the key recommendations from the 26 made in the report. Other recommendations deal with the regulation of Assitants in Nursing (AINs), the training of ENs, the accreditation and governance of nursing programs, and other peripheral issues. A noticeable absence from the report was any recommendation regarding postgraduate education in nursing which is an area that very much warrants a review. Overall, I was happy that the review was conducted and think that most of the recommendations are very reasonable. It is clear that there will be significant changes in nursing education in Australia, which will provide perioperative nursing with the opportunity to reassert itself as a priority specialty - if we are ready and able to do so.

\section{Reference}

Schwartz S. Educating the Nurse of the Future: Report of the Independent Review into Nursing Education. Canberra: Department of Health; 2019. Available from: www.health.gov.au/resources/ publications/educating-thenurse-of-the-future?utm source=miragenews\&utm medium=miragenews\&utm campaign=news. 